\title{
Retrospective analysis of transvaginal ultrasound-guided aspiration of simple ovarian cysts
}

\author{
Marta Kostrzewa ${ }^{A, C D}$, Agnieszka Zając ${ }^{B}$, Jacek Radosław Wilczyński ${ }^{C, E, F}$, Grzegorz Stachowiak ${ }^{A, B, E, F}$ \\ Polish Mother's Memorial Hospital Research Institute Department of Operative Gynecology and Gynecologic Oncology, Łódź, Poland \\ A - research concept and design; B - collection and/or assembly of data; C - data analysis and interpretation; \\ $D$ - writing the article; $E$ - critical revision of the article; $F$ - final approval of the article
}

Address for correspondence

Maria Kostrzewa

E-mail: kostrzewa.marta1@gmail.com

Funding sources

None declared

Conflict of interest

None declared

Received on November 1, 2017

Reviewed on July 18, 2018

Accepted on February 18, 2019

Published online on October 29, 2019

Cite as

Kostrzewa M, Zając A, Wilczyński JR, Stachowiak G.

Retrospective analysis of transvaginal ultrasound-

guided aspiration of simple ovarian cysts. Adv Clin Exp Med.

2019;28(11):1531-1535. doi:10.17219/acem/104549

DOI

10.17219/acem/104549

Copyright

Copyright by Author(s)

This is an article distributed under the terms of the

Creative Commons Attribution Non-Commercial License

(http://creativecommons.org/licenses/by-nc-nd/4.0/)

\begin{abstract}
Background. The widespread availability of ultrasonography means that transvaginal ultrasonography has become a routine procedure during gynecological examinations, even in asymptomatic patients. Nowadays the imaging technology offered by ultrasonography and tumor biomarkers give us an opportunity to implement transvaginal ultrasound-guided aspiration as a less radical treatment of simple ovarian cysts (SOC).

Objectives. The aim of the study was a retrospective evaluation of the diagnostic and therapeutic efficacy of transvaginal ultrasound-guided aspiration of SOC in postmenopausal and premenopausal patients.

Material and methods. A total of 84 women, divided into a premenopausal group (38/84) and a postmenopausal group (46/84), underwent transvaginal ultrasound-guided aspiration of small SOC (40-80 mm in diameter). Simple cysts were defined ultrasonographically according to the International Ovarian Tumor Analysis (IOTA) guidelines as cysts with negative risk of ovarian malignancy algorithm (ROMA) scores and CA125 levels. Simple ovarian cyst-related data was obtained from medical documentation (diagnostic tests, medical reproductive and surgical history, and clinical status during SOC aspiration). Follow-up data was collected by means of a telephone interview and medical database. The survey included questions focused on cyst recurrence during the 24-month period following the aspiration of $\mathrm{SOC}$.
\end{abstract}

Results. We had 100\% compatibility with ultrasound diagnosis and cytological examination of aspirated fluid. The cumulative rate of cyst recurrence among 84 patients was $20.2 \%$ (17/84). There was a higher percentage of cyst recurrence in the premenopausal group: $27 \%$ (10/38) vs 15.2\% (7/46) in the postmenopausal group, but the difference was not statistically significant (hazard ratio (HR) $=1.89,95 \%$ confidence interval $(95 \% \mathrm{Cl})=0.72-4.97 ; p=0.19)$. Recurrent cysts were treated with laparoscopic cystectomy, adnexectomy or a second aspiration in accordance with individual indications.

Conclusions. Ultrasound-guided aspiration of small ( $<80 \mathrm{~mm}$ ) adnexal SOC is a diagnostic and alternative therapeutic procedure, which allows cytological examination and may reduce the need for surgery, which is especially beneficial for women of reproductive age.

Key words: transvaginal ultrasound-guided aspiration, simple ovarian cysts, simple cyst recurrence 
The widespread use of medical ultrasonography, including transvaginal sonographic imaging, has become a routine procedure during gynecological examinations even in asymptomatic patients. One of its beneficial effects is frequent identification of adnexal masses, including simple ovarian cysts (SOC). Fortunately, the majority of these unilocular, anechoic, thin-walled cysts are benign in character. ${ }^{1}$ The management of SOC is dependent on the patient's age. Traditionally, due to the risk of malignancy, adnexal cysts or even palpable ovaries in postmenopausal women have been treated surgically, using either laparoscopy or laparotomy. Regarding premenopausal women, more than $66 \%$ of diagnosed ovarian cysts are functional. ${ }^{1,2}$ That is why the initial treatment of SOC in younger women starts with oral contraceptives, while laparoscopic cystectomy is only performed as second-line therapy. ${ }^{2}$

Nowadays, the imaging diagnostics offered by ultrasonographic technology and tumor biomarkers give us an opportunity to use transvaginal ultrasound-guided aspiration as a less radical treatment of SOC. This procedure is associated with a number of advantages, including fertility preservation, less risk of pelvic adhesions and lower surgery-related morbidity rates, while the lack of any histopathological examination may be perceived as the main disadvantage of this procedure.

The aim of our study was to retrospectively evaluate the diagnostic and therapeutic efficacy of transvaginal ultrasound-guided aspiration of SOC in post- and premenopausal patients.

\section{Material and methods}

The study involved 105 female patients who had been qualified for fine-needle aspiration of SOC at the Department of Gynecology and Gynecological Oncology of the Mother's Memorial Hospital Research Institute,
Łódź, Poland, in the years 2011-2015. During follow-up, contact was lost with 21 patients, who were therefore excluded from our study. Ultimately, the study involved 84 patients, who underwent follow-up ultrasonographic examination 24 months after the cyst aspiration procedure. The patients were divided into 2 groups: premenopausal $(\mathrm{n}=38)$ and postmenopausal $(\mathrm{n}=46)$.

Medical data on the SOC were collected from patient records stored in the hospital database. Each patient was told on discharge not to neglect control ultrasonographic examinations at the outpatient unit 3 and 6 months after the cyst aspiration. If the hospital database showed no cyst recurrence data during the 24-month time interval after the procedure, telephone enquiries with the patients were conducted for any complementary information.

A SOC was defined as qualified for aspiration when it was sonographically identified as a unilocular, anechoic, thin-walled tumor, without blood flow in a power Doppler (PD) examination, and with a $40-80 \mathrm{~mm}$ diameter. Additionally, all the patients had CA125 levels and risk of ovarian malignancy algorithm (ROMA) scores measured before aspiration and had no other gynecological disorders or lesions. They also had negative results on inflammation tests (white blood cell count (WBC)) and C-reactive protein (CRP)).

In order to avoid laparoscopic cystectomy, the patients in the premenopausal group were qualified for ultrasoundguided aspiration of a SOC if they had complained of periodic pelvic pains, which are especially characteristic of cysts over $50 \mathrm{~mm}$. Progressive cysts that occurred during a 3-6-month observation period despite oral contraceptive treatment were also included. The essential criteria for cyst aspiration included negative ROMA and serum CA125 levels. Additionally, 2 premenopausal patients with SOC between $40 \mathrm{~mm}$ and $50 \mathrm{~mm}$ in diameter were qualified for fine-needle aspiration because of cancerophobia $(2 / 38)$ (Table 1$)$.

Table 1. Characteristics of the study groups

\begin{tabular}{|c|c|c|c|}
\hline Variable & $\begin{array}{l}\text { Premenopausal } \\
\qquad n=38\end{array}$ & $\begin{array}{l}\text { Postmenopausal } \\
\qquad n=46\end{array}$ & $\mathrm{p}$-value \\
\hline $\begin{array}{l}\text { Age } \\
\text { Indications for FANC } \\
\text { periodic pelvic pain } \\
\text { enlargement or persistent simple cyst despite contraception } \\
\text { persistent over a year } \\
\text { cancerophobia }\end{array}$ & $\begin{array}{l}31.7 \pm 9.1 \\
30 \\
36 \\
- \\
2\end{array}$ & $\begin{array}{c}61.4 \pm 7.5 \\
10 \\
- \\
46 \\
-\end{array}$ & \\
\hline Mean diameter of the cyst [mm] & $62.3 \pm 21.2$ & $57.2 \pm 19.5$ & $>0.05$ \\
\hline Aspirated volume $[\mathrm{mL}]$ & $89.2 \pm 87.7$ & $89.5 \pm 113.9$ & $>0.05$ \\
\hline CA $125[\mathrm{IU} / \mathrm{mL}]$ & $11.5 \pm 7.35$ & $13.99 \pm 8.26$ & $>0.05$ \\
\hline Inflammatory cells in fluid (lymphocytes, neutrophils) & $3 / 38(7.9 \%)$ & $2 / 46(4.36 \%)$ & $>0.05$ \\
\hline Recurrence rate & $10 / 38(27 \%)$ & $7 / 46(15.2 \%)$ & $>0.05$ \\
\hline Second aspiration & $1 / 10(10 \%)$ & $2 / 7(28.5 \%)$ & $>0.05$ \\
\hline Laparoscopy or laparotomy in recurrence & $7 / 10(70 \%)$ & $4 / 7(57.1 \%)$ & $>0.05$ \\
\hline Positive microbiological examination & $13 / 38(34.2 \%)$ & $16 / 46(34.8 \%)$ & $>0.05$ \\
\hline
\end{tabular}


In the postmenopausal group, the presence of an asymptomatic SOC with a diameter over $40 \mathrm{~mm}$ and normal ROMA and CA125 levels, persistent for over a year, qualified the patient for ovarian aspiration instead of laparoscopic cystectomy. Ten of the postmenopausal patients were qualified for ultrasound-guided aspiration because of mild pelvic pains.

Women with pelvic pain were examined in order to exclude any other causes of the pain before ultrasound-guided transvaginal aspiration of the SOC was finally decided. All the patients provided their informed consent before the procedure and had transvaginal ultrasonographic follow-ups 3, 6, 12, and 24 months after the procedure, performed either at an outpatient clinic or on a subsequent admission to hospital. Recurrence was defined as the presence of an adnexal cyst on the same ovary with the largest diameter of $30 \mathrm{~mm}$ or greater and still persistent at 3-6 subsequent months in premenopausal women (to distinguish between the formation of a new functional cyst and a true recurrence) and a cyst larger than $10 \mathrm{~mm}$ in postmenopausal women. Similar criteria for ovarian cyst recurrence are reported in the literature. ${ }^{3}$

The mean age in the premenopausal and postmenopausal groups was 31.7 years and 61.4 years, respectively. The right ovary was affected in $54.76 \%$ of the patients $(46 / 84)$. In 2 cases, cysts were located bilaterally, and in $42.8 \%$ of the cases (36/84), they were left-sided (Table 1 ).

\section{Procedure}

The ultrasound-guided aspiration procedures were performed by a gynecologist trained in gynecological surgery, using a 5-7 MHz intravaginal probe. No anesthesia was administered in $25 / 84$ of the patients (29.7\%), while short intravenous anesthesia was requested by 59 of the patients (70.2\%). After disinfecting the vagina, a 22-gauge needle attached to a vaginal probe was used for the aspiration. It was ejected when the probe was fixed and the SOC was clearly visualized. The needle was sonographically guided directly into the SOC and all the fluid was aspirated into a syringe. The fluid aspirated from the cyst was then sent for cytological and microbiological examination. The whole procedure took approx. 3-4 min, without any complications for any of the patients either during or after the intervention.

\section{Statistical analysis}

The $\chi^{2}$ test was used for non-parametric values, while parametric values were compared with Student's t-test. The cyst recurrence rate was compared between groups, using the Kaplan-Meier (K-M) survival estimator. Diagrams were plotted showing the survival rate without ovarian cyst recurrence during 24 months. The diagrams were compared using the log-rank test and Cox's proportional hazard models to calculate the relative chance for cyst recurrence during the period evaluated. The level of statistical significance was set at $\mathrm{p}<0.05$. The statistical analysis was carried out using STATISTICA v. 12 software (StatSoft Inc., Tulsa, USA).

\section{Results}

The cytological examinations did not reveal any atypical cells in aspirated fluid. Inflammation cells were found in 5 cases; however, this did not correlate either with the patients' symptoms or the results of the microbiological examinations. In addition, in 29/84 patients (34.5\%), the results of the microbiological examinations were positive. Positive microbiological test results revealed Grampositive Staphylococci and Streptococci: Staphylococcus epidermidis (13/29; 44.8\%), S. kloosii (5/29; $17.2 \%)$ and Streptococcus agalactiae (11/29; 37.9\%). It is worth pointing out that all of the cultured bacteria belonged to typical bacterial flora of the human vagina. All the patients had normal WBC counts and negative CRP levels, so no antibiotic therapy was needed.

The cumulative rate of cyst recurrence among the 84 patients was $20.2 \%$ (17/84). There was higher percentage of cyst recurrence in the premenopausal group than in the postmenopausal group $(27 \% 10 / 38, \mathrm{~K}-\mathrm{M}$ estimate $=0.73) \mathrm{vs}$ $15.2 \%(7 / 46, \mathrm{~K}-\mathrm{M}$ estimate $=0.85))$, but the difference was statistically insignificant: hazard ratio $(\mathrm{HR})=1.89$ (95\% confidence interval $(95 \% \mathrm{CI})=0.72-4.97 ; \mathrm{p}=0.19)$ (see Fig. 1).

In the 10 cases of cyst recurrence among the premenopausal patients, 6 laparoscopic cystectomies, 1 adnexectomy and $12^{\text {nd }}$ aspiration were performed; 2 patients declined further interventions during the follow-up period. In the 7 cases of recurrence in the postmenopausal group, 2 patients underwent $2^{\text {nd }}$ aspirations and 4 had adnexectomies; 1 patient declined further interventions during the follow-up period. Second aspirations were performed when the patient did not agree to laparoscopy. Histopathological studies, performed after laparoscopic cystectomy and adnexectomy, revealed the presence of SOC.

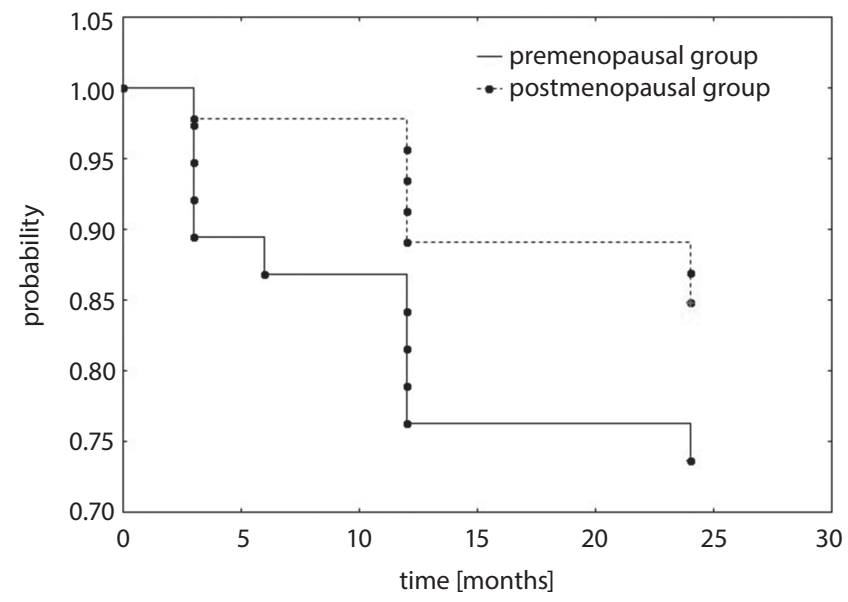

Fig. 1. Probability of survival without recurrence of the ovarian cyst 


\section{Discussion}

When applied hastily, transvaginal ultrasound-guided aspiration of an ovarian lesion may be perceived as a rather controversial approach, not only from the diagnostic but also from the therapeutic point of view. First, it may pose a risk of spreading atypical cells from an unsuspected malignant tumor. Up to $24 \%$ of ovarian tumors in premenopausal women and up to $60 \%$ in postmenopausal women are malignant. ${ }^{4}$ Second, aspiration is thought to be ineffective due to continued fluid production by the cyst wall. In the literature, the incidence rates of cyst recurrence after aspiration range $11-75 \%$, but in some studies endometrial and dermoid cysts were included. ${ }^{3,5-8}$

Fortunately, a SOC is quite easy to diagnose with ultrasonography, ROMA and CA125 biomarkers. Moreover, malignant transformation is a rather rare process in SOC. In our study, the initial sonographic diagnosis of SOC was $100 \%$ compatible with the results of the cytological examinations. Gupta et al. showed high specificity (98.0\%) and sensitivity (85.7\%) levels for the diagnostics of ovarian/adnexal lesion malignancy with fine-needle aspiration, with a positive predictive value of $97.7 \%$, a negative predictive value of $87.7 \%$ and accuracy of $92.0 \%$. They compared cytology results from fine-needle aspiration cytology (FNAC) with the results of histopathological studies. ${ }^{9}$ Similarly, Ray et al. revealed high concordance between the results of FNAC and histopathological examinations: The sensitivity of cytological diagnosis was $83 \%$, the specificity was $97 \%$ and its accuracy was $93 \% .^{10}$

Preoperative cytodiagnostics of ovarian masses were conducted by Pal et al., who carried out ultrasound-guided FNAC in 70 cases. Their cytological examinations reached over $93 \%$ accuracy in relation to histopathological diagnoses; the sensitivity and specificity rates of their cytological examinations were $95.23 \%$ and $95.83 \%$, respectively. Based on their results, the authors stated that ultrasoundguided FNAC was a quick, economic and safe procedure in diagnosing ovarian masses with brilliant accuracy. ${ }^{11}$ Fine-needle aspiration of ovarian masses was also found to be highly specific and moderately sensitive for the detection of ovarian malignancies by Zhou et al. ${ }^{12}$ Nagamine et al. confirmed the high sensitivity (75\%) and specificity (100\%) of FNAC in the identification of malignant lesions in a study involving intraoperative FNAC before ovarian tumor excision. ${ }^{13}$

Lokich et al. evaluated the use of ROMA as a diagnostic tool in women with adnexal masses, identifying low malignancy risk cases which could safely be treated with conservative, non-surgical methods. The use of ROMA seems to be valuable particularly in premenopausal women, where the incidence of epithelial ovarian cancer is low, while cases of benign disease are numerous. In conclusion, they asserted that ROMA in conjunction with detailed ultrasound imaging could safely identify women with lowmalignancy adnexal masses, appropriate for conservative therapy. Their results confirmed that nonsurgical management should be considered for SOC identified with ultrasonography and negative ROMA and CA125 levels. ${ }^{14}$

The patients qualified for fine-needle aspiration at our department presented SOC under $80 \mathrm{~mm}$ in diameter. Cysts with diameters above $80 \mathrm{~mm}$ were qualified for surgery. According to García-Tejedor et al., a higher risk of recurrence was found for cysts larger than $70 \mathrm{~mm}$ (odds ratio $(\mathrm{OR})=4.2 ; 95 \% \mathrm{CI}=1.2-14.1){ }^{3}$ Yamamoto et al. estimated a higher risk of malignancy in tumors $\geq 70 \mathrm{~mm}$ in size. They found the Risk of Malignancy Index (RMI) including parameters like menopausal or premenopausal status, ultrasonographic findings, CA125 level, and tumor size to be $91.0 \%$ specific with a sensitivity of $86.6 \%$ in preoperative evaluation of adnexal masses regarding malignancy. ${ }^{15}$

According to a statement of the Society of Radiologists in Ultrasound from 2010, in premenopausal women, cysts $>3 \mathrm{~cm}$ and $\leq 5 \mathrm{~cm}$ should be described in imaging impressions as almost certainly benign and not in need of follow-up. ${ }^{16}$ The major ultrasonographic features of ovarian malignancy, described by the IOTA Group and including solid or complex ovarian tumors, pathological velocity etc., also account for the size of the tumor. The mean diameter of benign adnexal masses in the IOTA study was $63 \mathrm{~mm}$, while the mean diameter of malignant tumors was $100 \mathrm{~mm}$. However, ultrasonographic findings of a benign character of a tumor are not always confirmed in histopathological evaluations. In the IOTA study, 2 patients out of 1,066 had borderline tumors, although no signs of malignancy were revealed in ultrasonography. This is, in our opinion, why patients with SOC, regardless of their age, should have, as a minimum, ultrasonographic examination in their follow-up. ${ }^{17}$

García-Tejedor et al. showed a 39\% (34/87) recurrence rate of SOC in their follow-up examinations. ${ }^{4}$ Similar results, although in a rather small study group, were obtained by Nikolaou et al., who observed a $35.2 \%(n=12)$ recurrence rate after transvaginal ultrasound-guided aspiration of serous ovarian cysts. ${ }^{8}$ In our study, the general recurrence rate was considerably lower: $20.2 \%$ (17/84); it was $27 \%$ in the premenopausal group and $15.2 \%$ in the postmenopausal group. Probably this difference depends on size of the tumors. In our study, only small ovarian cysts, i.e., $\leq 80 \mathrm{~mm}$, were qualified for aspiration.

In general, the recurrence rate of benign ovarian cysts in our previous study was 19\% (excluding endometriomas) in premenopausal women who had undergone laparoscopic cystectomies, monitored during a 24-month follow-up. Comparing our recurrence rates after fine-needle aspiration in the premenopausal group with those following surgical treatment (27\% vs $19 \%$ ), it becomes fairly obvious that transvaginal ultrasound-guided aspiration of SOC is a procedure worth considering in young women to avoid surgery-related ovarian reserve reduction, thus preserving fertility, especially in cases of subsequent recurrence. ${ }^{18}$ 
Our study demonstrated insignificantly lower recurrence rates of SOC in the postmenopausal women vs the premenopausal group ( $15 \%$ vs $27 \%$, respectively). This shows that fine-needle aspiration may, in reasonable cases, be considered a less radical management method for SOC in postmenopausal patients. In some studies, even conservative management of SOC in postmenopausal women is taken into account, particularly for cysts $\leq 50 \mathrm{~mm}$. Spontaneous disappearance of ovarian cysts $\leq 50 \mathrm{~mm}$ in postmenopausal patients has been reported by many authors, with prevalence rates up to $50 \%(8.3-50 \%) .{ }^{19-21}$ The previously reported recurrence of SOC and sactosalpinx in postmenopausal women after FNAC was $25 \%{ }^{22}$

Better results after the procedure with lower recurrence rates (15\%) of SOC were achieved in premenopausal women on oral contraceptives by Koutlaki et al. However, the researchers noted much higher recurrence rates in postmenopausal groups $(10 / 17(58.9 \%)) .{ }^{23}$ Our study results confirm that transvaginal fine-needle aspiration of SOC is a reliable alternative to surgery, demonstrating many advantages, such as excellent tolerance, low risk and low recurrence rates, especially in premenopausal women.

Gupta et al. also published promising results performing percutaneous aspiration and subsequent methotrexate injection into SOC and endometriomas. In follow-up ultrasonography in their study, cysts had disappeared in 120 patients (90.9\%) and persisted in 12 patients (9.1\%). ${ }^{24}$

Transvaginal ultrasound-guided aspiration of SOC, performed in properly qualified patients, may reduce to a minimum the risk of procedure-related malignant transformation of the lesions. Furthermore, the low recurrence ratio, comparable to that following laparoscopic cystectomies, shows that it may be considered secondstage procedure after conservative management of SOC or regarded as a less radical treatment in difficult recurrent cases of SOC with negative CA125 levels and ROMA scores. Transvaginal ultrasound-guided aspiration can eliminate risk of pelvic adhesions or morbidity due to surgery, and can preserve fertility, which can be especially important in young women.

Summing up, transvaginal ultrasound-guided aspiration of SOC should be considered an alternative method for the treatment of selected cases of adnexal cysts in women of all ages.

\section{References}

1. Timmerman D, Valentin L, Bourne $T H$, Collins WP, Verrelst $H$, Vergote l; International Ovarian Tumor Analysis (IOTA) Group. Terms, definitions and measurements to describe the sonographic features of adnexal tumors: A consensus opinion from the International Ovarian Tumor Analysis (IOTA) Group. Ultrasound Obstet Gynecol. 2000; 16(5):500-505.

2. Spaczyński M, Bidziński M, Basta A, et al. Rekomendacje PTG dotyczące postępowania w guzach niezłośliwych i raku jajnika. Ginek Onkol. 2006;4(2):131-135.
3. Gillis CR, Hole DJ, Still RM, Davis J, Kaye SB. Medical audit, cancer registration, and survival in ovarian cancer. Lancet. 1991;337(8741): 611-612.

4. García-Tejedor A, Castellarnau M, Burdio F, et al. Ultrasound-guided aspiration of adnexal cysts with a low risk of malignancy: Is it a recommendable option? J Ultrasound Med. 2015;34(6):985-991.

5. Duke D, Colville J, Keeling A, Broe D, Fotheringham T, Lee MJ. Transvaginal aspiration of ovarian cysts: Long-term follow-up. CardiovasC Intervent Radiol. 2006;29(3):401-405.

6. Petrovic N, Arko D, Lovrec VG, Takac I. Ultrasound-guided aspiration in pathological adnexal processes. Eur J Obstet Gynecol Reprod Biol. 2002;104(1):52-57.

7. Troiano RN, Taylor KJ. Sonographically guided therapeutic aspiration of benign-appearing ovarian cysts and endometriomas. AJR Am J Roentgenol. 1998;171(6):1601-1605.

8. Nikolaou M, Adonakis G, Zyli P, et al. Transvaginal ultrasound-guided aspiration of benign ovarian cysts. J Obstet Gynaecol. 2014;34(4): 332-335.

9. Gupta N, Rajwanshi A, Dhaliwal LK, et al. Fine needle aspiration cytology in ovarian lesions: An institutional experience of 584 cases. Cytopathology. 2012;23(5):300-307.

10. Ray S, Gangopadhyay M, Bandyopadhyay A, Majumdar K, Chaudhury N. USG guided FNAC of ovarian mass lesions: A cyto-histopathological correlation, with emphasis on its role in pre-operative management guidelines. J Turk Ger Gynecol Assoc. 2014;15(1):6-12.

11. Pal S, Chakrabarti S, Deuoghuria D, Phukan JP, Sinha A, Mondal PK. Evaluation of ultrasound-guided fine-needle aspiration cytology of ovarian masses with histopathological correlation. Acta Cytol. 2015; 59(2):149-155.

12. Zhou AG, Levinson KL, Rosenthal DL, van den Bussche CJ. Performance of ovarian cyst fluid fine-needle aspiration cytology. Cancer Cytopathol. 2018;126(2):112-121.

13. Nagamine K, Kondo J, Kaneshiro R, Tauchi-Nishi P, Terada K. Ovarian needle aspiration in the diagnosis and management of ovarian masses. J Gynecol Oncol. 2017;28(4):1-10.

14. Lokich E, Palisoula M, Romanoa N, et al. Assessing the risk of ovarian malignancy algorithm for the conservative management of women with a pelvic mass. Gynecol Oncol. 2015;139(2):248-252.

15. Yamamoto $Y$, Yamada R, Oguri H, Maeda N, Fukaya T. Comparison of four malignancy risk indices in the preoperative evaluation of patients with pelvic masses. Eur J Obstet Gynecol Reprod Biol. 2009; 144(2):163-167.

16. Levine D, Brown DL, Andreotti RF, et al. Management of asymptomatic ovarian and other adnexal cyst imaged at US: Society of Radiologists in Ultrasound Consensus Conference Statement. Radiology. 2010;256(3):943-954.

17. Timmerman D, Testa AC, Bourne T, et al; International Ovarian Tumor Analysis Group. Logistic regression model to distinguish between the benign and malignant adnexal mass before surgery: A multicenter study by the International Ovarian Tumor Analysis Group. J Clin Oncol. 2005;23(34):8794-8801.

18. Kostrzewa M, Stachowiak G, Żyła M, et al. Women's fertility after laparoscopic cystectomy of endometrioma and other benign ovarian tumors: A 24-month follow-up retrospective study. Neuro Endocrinol Lett. 2016;37(4):101-106.

19. Wolf SI, Gosink BB, Feldesman MR, et al. Prevalence of simple adnexal cysts in postmenopausal women. Radiology. 1991;180(1):65-71.

20. Andolf $E$, Jörgensen C. Simple adnexal cysts diagnosed by ultrasound in postmenopausal women. J Clin Ultrasound. 1988;16(5):301-303.

21. Aubert JM, Rombaut C, Argacha P, et al. Simple adnexal cysts in postmenopausal women: Conservative management. Maturitas. 1998; 30(1):51-54.

22. Jędrzejczyk S, Stachowiak G, Zając A, et al. Fine needle aspiration in the treatment of adnexal cysts in women in menopausal period. Prz Menopauzalny. 2007;6(2):90-95.

23. Koutlaki N, Nikas I, Dimitraki M, Grapsas X, Psillaki A, Mandratz J. Transvaginal aspiration of ovarian cysts: Our experience over 121 cases. Minim Invasive Ther Allied Technol. 2010;20(3):155-159.

24. Gupta P, Huria A. Management of ovarian cysts with percutaneous aspiration and methotrexate injection. Niger Med J. 2016;57(1):19-23. 